Mitsuhiro Abe', Kenji Tsushima², Daisuke Ishii', Kohei Shikano', Keiichiro Yoshioka', Masashi Sakayori', Masaki Suzuki', Yasutaka Hirasawa', ${ }^{1}$, Tsukasa Ishiwata', Takeshi Kawasaki', Jun Ikari', Jiro Terada' ${ }^{1,2}$, Koichiro Tatsumi ${ }^{1}$

'Department of Respirology, Graduate School of Medicine, Chiba University, Chiba, Japan

${ }^{2}$ Department of Pulmonary Medicine, International University of Health and Welfare, School of Medicine, Narita-city, Japan

\title{
Risk factors for acute exacerbation following bronchoalveolar lavage in patients with suspected idiopathic pulmonary fibrosis: A retrospective cohort study
}

\begin{abstract}
Introduction: Bronchoalveolar lavage (BAL) is useful for diagnosing diffuse lung disease and excluding other conditions. However, acute exacerbations (AEs) are recognized as important complications of BAL in patients with idiopathic pulmonary fibrosis (IPF). This study aimed to identify risk factors for BAL-induced AEs in patients with IPF.

Material and methods: We retrospectively analyzed the data of 155 patients with suspected IPF who had undergone BAL between January 2013 and December 2018. BAL-related AE was defined as the development of AE within 30 days after the procedure. We compared clinical features and parameters between patients with $A E$ (AE group) and without AE (non-AE group). We also reviewed the relevant reported literature.

Results: Among the 155 patients, 5 (3.2\%) developed AE within 30 days after BAL. The average duration from BAL to AE onset was 7.8 days (2-16 days). Results from the univariate analysis revealed $\mathrm{PaO}_{2}<75 \mathrm{~mm} \mathrm{Hg}(\mathrm{p}=0.036)$, neutrophil content in $\mathrm{BAL}$ $\geq 7 \%(p=0.0061), \% D_{L C 0}<50 \%(p=0.019)$, Gender-Age-Physiology (GAP) stage III $(p=0.034)$, and BAL recovery rates $<$ $30 \%(p<0.001)$ as significant risk factors for post-BAL AE. All five patients who developed AE recovered and were discharged. Conclusions: Disease severity, high neutrophil levels in BAL, and poor BAL recovery rates may be risk factors for BAL-induced AEs.
\end{abstract}

Key words: bronchoalveolar lavage, C-reactive protein, idiopathic pulmonary fibrosis, interstitial lung disease, risk factor

Adv Respir Med. 2021; 89: 101-109

\section{Introduction}

Bronchoalveolar lavage (BAL) is a standard tool for the diagnostic and prognostic evaluation of diffuse lung diseases [1-3]. BAL is useful for differentiating idiopathic pulmonary fibrosis (IPF) from other fibrosing lung diseases, such as non-specific interstitial pneumonia (NSIP), chronic hypersensitivity pneumonia (CHP), and interstitial pneumonia due to collagen and vasculitis disease. Ohshimo et al. [4] reported that $8 \%$ of patients with a usual interstitial pneumonia (UIP) pattern on high-resolution computed tomography (HRCT) might have BAL findings suggestive of such an alternative diagnosis.

\section{Clinical rationale for the study}

The American Thoracic Society (ATS), European Respiratory Society (ERS), Japanese Respiratory Society (JRS), and Latin American Thoracic Society (ALAT) 2011 guidelines advocated that the most important application of BAL when evaluating patients with suspected IPF is CHP exclusion; prominent lymphocytosis ( $>40 \%$ ) should suggest CHP [5]. The ATS/JRS/ALAT 2020 guidelines on CHP by Raghu et al. described the importance of BAL in diagnosing CHP [6]. Per the ATS/ERS/JRS/ALAT 2018 guidelines [7], BAL is not recommended for patients with a UIP pattern because of the 


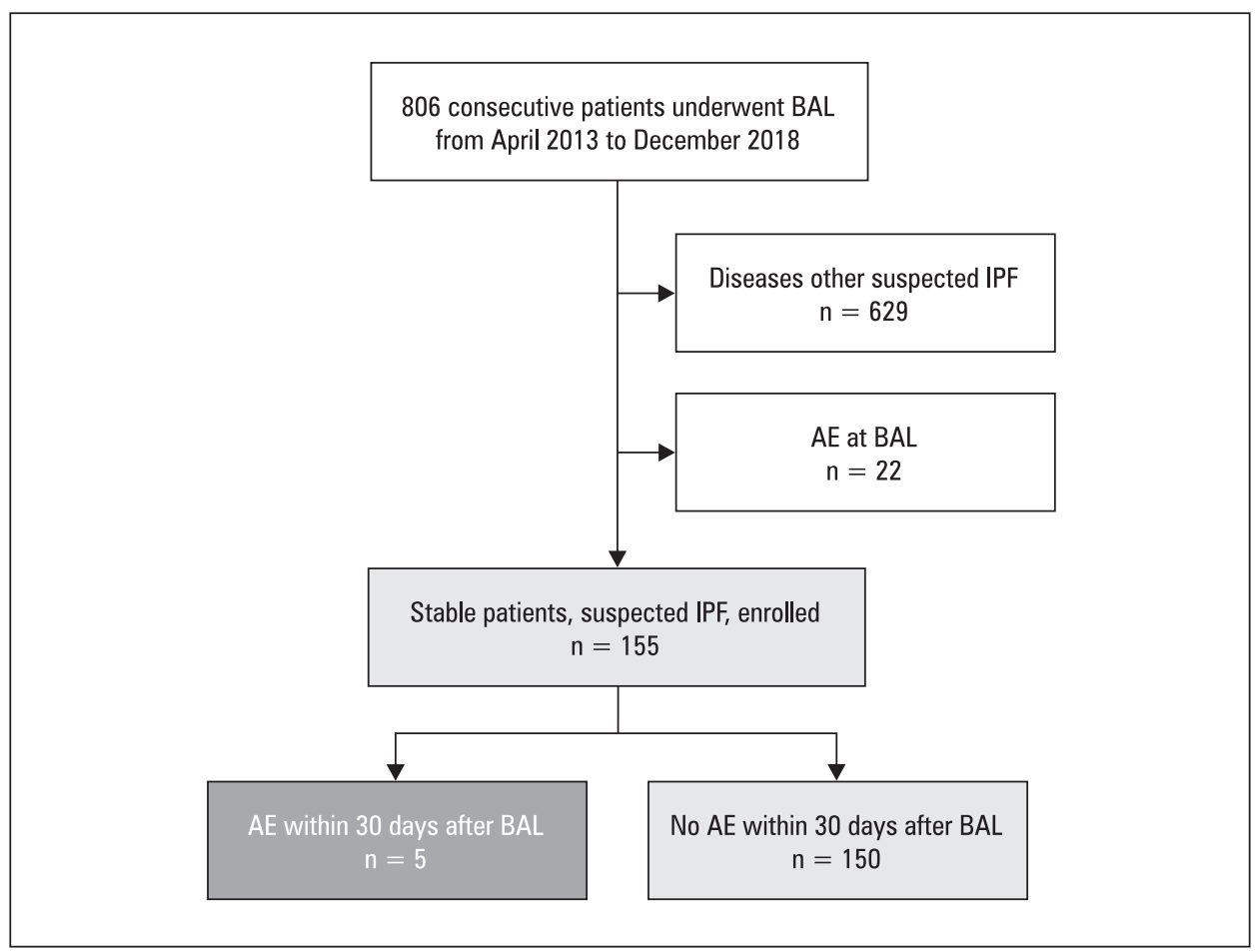

Figure 1. Study flow chart. We identified 806 consecutive patients who underwent bronchoalveolar lavage (BAL) from April 2013 to December 2018. Of them, 629 patients were diagnosed with diseases other than suspected IPF; therefore, they were excluded from the analysis. Additional 22 patients with acute exacerbation were excluded. Therefore, 155 individuals were enrolled in the study. Five patients developed acute exacerbation within 30 days after the BAL procedure. BAL — bronchoalveolar lavage; IPF — idiopathic pulmonary fibrosis; AE — acute exacerbation

risk of acute exacerbation (AE) of IPF (AE-IPF) [8, 9]. Sakamoto et al. [8] reviewed 12 cases of BAL-induced AE-IPF and found that functional impairment or active inflammation may be risk factors for BAL-induced AE. Only patient factors were reported; no risk factors were evaluated for bronchoscopic procedures.

Therefore, this study aimed to evaluate the previously proposed risk factors for IPF-AE after BAL and identify other novel risk factors for IPFAE after BAL.

\section{Material and methods}

Our single-center retrospective study was approved by our human ethics committee (protocol number 2083). We obtained informed consent with an opt-out option. The study was conducted in accordance with the ethical principles of the 1964 Declaration of Helsinki and subsequent amendments.

\section{Patients}

Between January 2013 and December 2018, 806 consecutive patients underwent BAL at our hospital. Among them, 629 subjects were suspected of having non-IPF diseases, including an "alter- native diagnosis" per the 2018 ATS/ERS/JRS/ALAT statement [7]; drug-induced lung injury; interstitial lung disease with collagen vascular disease; acute respiratory distress syndrome; etc.; therefore, they were excluded from our analysis. Twenty-two patients suspected of having $\mathrm{AE}$ at the time of BAL were excluded. Finally, 155 individuals suspected of having stable (non-exacerbation) IPF were analyzed (Figure 1). Then, these patients underwent their first BAL procedure.

\section{UIP pattern on HRCT}

Radiological diagnosis on HRCT (UIP, probable UIP, and indeterminate for UIP) was determined per the 2018 ATS/ERS/JRS/ALAT guidelines [7]. After discussion between two respiratory specialists, the HRCT pattern was classified as either UIP ( $n=68)$, probable UIP ( $n=57$ ), or indeterminate for UIP $(\mathrm{n}=30)$ (Table 1).

\section{IPF confidence}

Surgical lung biopsy was performed in eight cases. The final diagnoses were expressed using the four diagnostic confirmation levels proposed by Ryerson et al. [10]: a "confident diagnosis" meets $\geq 90 \%$ of the guidelines, a "high-confidence diagnosis" meets 70-89\%, a "low-confidence di- 
Table 1. Baseline characteristics

\begin{tabular}{|c|c|}
\hline & $n=155$ \\
\hline Age [years] & $68.6 \pm 7.0$ \\
\hline Male, n [\%] & $110(70 \%)$ \\
\hline Smoker, n [\%] & $113(72 \%)$ \\
\hline $\begin{array}{l}\text { HRCT diagnosis } \\
\text { [UIP/probable UIP/indeterminate for UIP] }\end{array}$ & $68 / 57 / 30$ \\
\hline $\mathrm{PaO}_{2}[\mathrm{~mm} \mathrm{Hg}]$ & $80.5 \pm 12.5$ \\
\hline $\mathrm{KL}-6$ [U/mL] & $1353 \pm 1200$ \\
\hline CRP [mg/dL] & $0.4 \pm 1.0$ \\
\hline \%FVC [\%] & $75.6 \pm 18.3$ \\
\hline$\% D_{\text {LCO }}[\%]$ & $66.9 \pm 23.3$ \\
\hline GAP stage $[\mathrm{I} / \mathrm{II} / \mathrm{III}]$ & $87 / 54 / 14$ \\
\hline $\begin{array}{l}\text { Confidence level of IPF [confident/high } \\
\text { confidence/low confidence/unclassifiable] }\end{array}$ & 70/49/20/16 \\
\hline \multicolumn{2}{|c|}{$\begin{array}{l}\text { Data are expressed as mean } \pm \text { standard deviation. HRCT }- \text { high-resolutio } \\
\text { computed tomography; UIP - usual interstitial pneumonia; KL- } 6 \text { - Krebs vo } \\
\text { den Lungen-6; CRP - C-reactive protein; FVC - forced vital capacity; } D_{\text {Lco }} \\
\text { diffusing capacity of the lungs for carbon monoxide; GAP stage - Gender-Age } \\
\text { Physiology stage; IPF - idiopathic pulmonary fibrosis }\end{array}$} \\
\hline
\end{tabular}

agnosis" meets 51-69\%, and an "unclassifiable diagnosis" meets $<50 \%$ (Table 1 ).

\section{Gender-age-physiology stage}

Gender-Age-Physiology (GAP) stages were calculated per the criteria reported by Ley et al. [11]: sex (female, 0 points; male, 1 point), age ( $\leq$ 60 years, 0 points; $61-65$ years, 1 point; $>65$, 2 points), predicted forced vital capacity (\%FVC) (> 75\%, 0 points; $50-75 \%$, 1 point; $<50 \%$, 2 points), and predicted diffusing capacity for carbon monoxide $\left(\% \mathrm{D}_{\mathrm{LCO}}\right)$ (> 55\%, 0 points; $36-55 \%, 1$ point; $\leq 35 \%$, 2 points; cannot obtain $\mathrm{D}_{\mathrm{LCO}}, 3$ points). The patients were divided into the following GAP stages based on their total GAP score: I ( $0-3$ points), II (4-5 points), and III (6-8 points).

\section{BAL procedures}

BAL procedures were performed using a flexible bronchoscope with a 5.9-mm outer diameter (BF-1TQ290 or BF-6C260; Olympus Corporation, Tokyo, Japan) under intravenous anesthesia. Sterile saline $(0.9 \% \mathrm{NaCl})$ at room temperature was instilled through the bronchoscope. Per the commonly used methodology in Japan, the total instilled volume of saline was $150 \mathrm{~mL}$ (50 $\mathrm{mL} \times 3$ times). As the lavage site, 127 cases were in the middle lobe or lingula, and 28 were at other sites. The lavage site was determined by considering the presence of the interstitial shadow. Transbronchial lung biopsy (TBLB) was performed after BAL in 131 cases. We did not perform transbronchial lung cryobiopsy.

\section{Diagnosis of BAL-induced AE-IPF (AE and non-AE groups)}

The patients were diagnosed with AE-IPF if they met the following criteria established by Collard [12]: (1) previous or concurrent IPF diagnosis, (2) acute worsening or development of dyspnea (typically of 1-month duration), (3) computed tomography with new bilateral groundglass opacity and/or consolidation superimposed on a background pattern consistent with a UIP pattern that appeared as new shadows on the BAL site and the opposite lung field, and (4) deterioration not fully explained by cardiac failure or fluid overload. BAL-induced AE-IPF was defined as AEIPF occurring within 30 days post-BAL procedure (AE group). The patients who did not develop AE within 30 days after the BAL procedure were included in the non-AE group.

\section{Statistical analysis}

Clinical data are expressed as mean \pm standard deviation. We compared the $\mathrm{AE}$ and nonAE groups using the Mann-Whitney $U$ test for continuous variables and Fisher's exact test for categorical variables. Youden's index and receiver operating characteristic curve analysis were used to identify parameters affecting $\mathrm{AE}$ within 30 days post-BAL (Figure 2). Univariate logistic regression analyses were used to identify factors affecting BAL-induced AE-IPF. All statistical analyses were performed using EZR (Saitama Medical Center, Jichi Medical University, Saitama, Japan) [13]. Statistical significance was defined as $\mathrm{p}<0.05$.

\section{Previous BAL-induced AE-IPF reports}

We searched the literature to identify previous BAL-induced AE-IPF reports published from 1977 to 2019. We used PubMed (https://www.ncbi. nlm.nih.gov/pubmed) for English reports and J-STAGE (https://www.jstage.jst.go.jp) for English and Japanese reports.

\section{Patient and public involvement}

The patients were not involved in the design, recruitment, or conduction of the studies included in this analysis.

\section{Results}

Table 1 shows the baseline characteristics of the 155 patients. According to HRCT, 68 patients exhibited the "UIP pattern," 57 showed a "proba- 


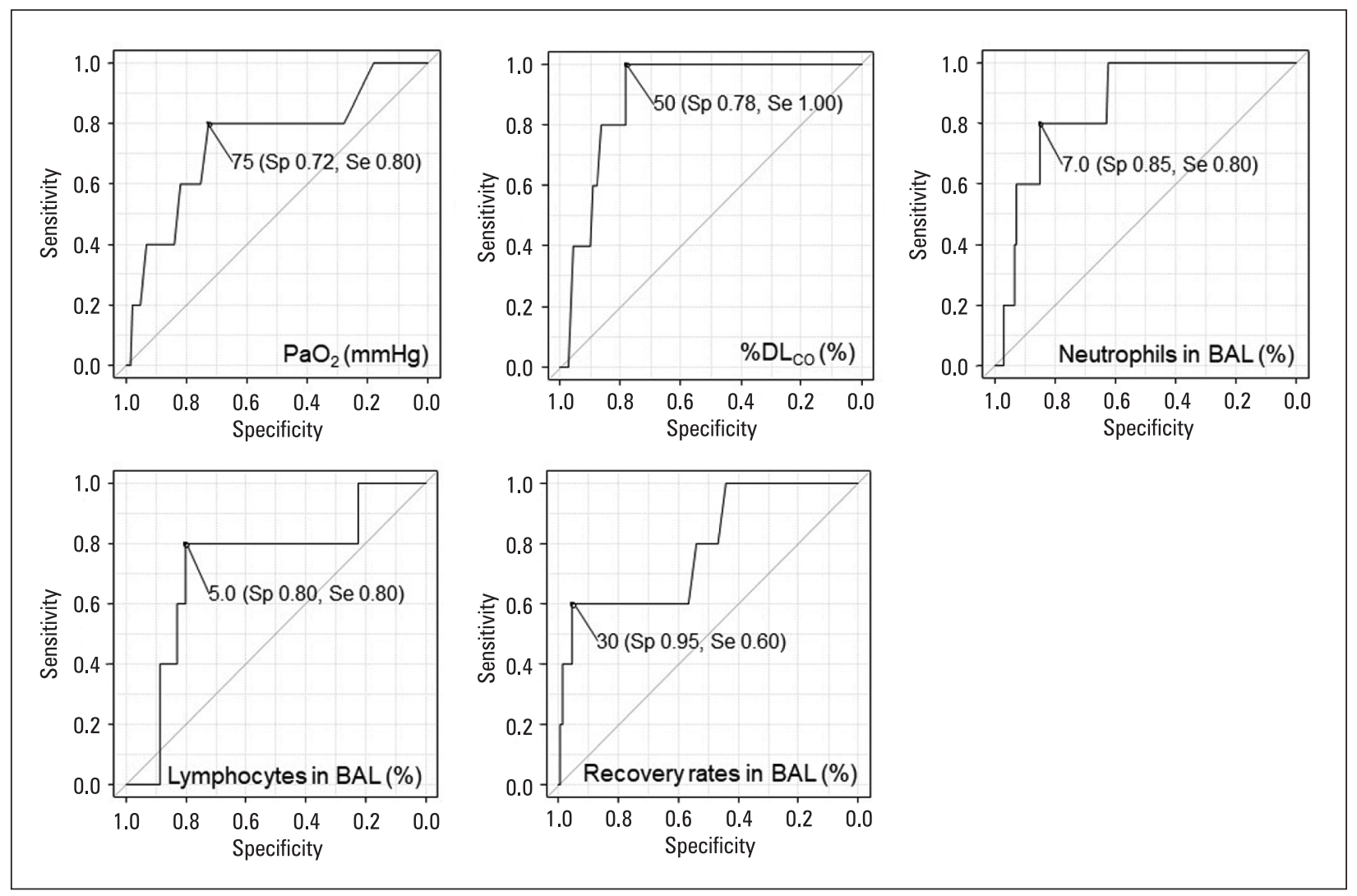

Figure 2. Receiver operating characteristic curve analysis was performed to obtain the parameters affecting acute exacerbation within 30 days of BAL. Sp — specificity; $\mathrm{Se}$ - sensitivity; $\mathrm{PaO}_{2}$ — partial pressure of oxygen; $\mathrm{D}_{\mathrm{Lco}}$ — diffusing capacity for carbon monoxide; $\mathrm{BAL}$ — bronchoalveolar lavage

ble UIP pattern", and 30 were considered "indeterminate for UIP" [7]. The GAP stage was relatively mild [9]. Specifically, 87 patients were classified as stage I, 54 as stage II, and 14 as stage III.

Thirty-one patients had $>25 \%$ of lymphocytes in BAL fluid, suggesting the presence of non-IPF diseases, such as CHP and NSIP. One of these patients was diagnosed with NSIP based on surgical lung biopsy. The other 30 subjects did not undergo surgical lung biopsy. Using the Ryerson method, the 155 patients were classified into four groups according to IPF confidence level (confident, 70; high confidence, 49; low confidence, 20; unclassifiable, 16; Table 1).

The median AE-free survival after bronchoscopy was 1057 days (range: 3-2263 days). Among the 155 patients, 19 (12\%) developed AE, and $25(16 \%)$ died within the study period. The causes of death were $\operatorname{AE}(n=6)$; respiratory failure $(n=15)$; and other causes $(n=4)$, including colon cancer $(n=$ 1), ovarian cancer $(n=1)$, liver failure due to viral hepatitis $(n=1)$, and heart failure $(n=1)$. The overall $\mathrm{AE}$ frequency throughout the whole observation period was $0.36 \%$ per 30 days and $4.4 \%$ per year.
Table 2 shows the clinical data of the five BAL-induced AE-IPF patients and non-AE patients. All five AE-IPF subjects were male, were past smokers, exhibited a UIP pattern on HRCT, and had confident IPF. Although partial pressure of oxygen $\left(\mathrm{PaO}_{2}\right)(\mathrm{p}=0.062)$ and \%FVC $(\mathrm{p}=0.36)$ were not significantly different between the two groups, $\% \mathrm{D}_{\mathrm{LCO}}$ was significantly lower in the $\mathrm{AE}$ group than in the non-AE group $(\mathrm{p}=0.0074)$. Additionally, the GAP stage was significantly more severe in the AE group than in the non-AE group $(p=0.0086)$. Further, the BAL recovery rate was significantly lower in the AE group than in the non-AE group ( $p=0.029)$. There was no significant difference in the lymphocyte fraction in BAL fluid between the two groups ( $p=0.086$ ), but the neutrophil fraction was significantly higher in the $\mathrm{AE}$ group than in the non-AE group $(\mathrm{p}=0.0059)$.

Univariate logistic regression analysis results revealed that $\mathrm{PaO}_{2}<75 \mathrm{~mm} \mathrm{Hg}(\mathrm{p}=0.036)$, neutrophils in BAL $\geq 7 \%(\mathrm{p}=0.0061), \% \mathrm{D}_{\mathrm{LCO}}<$ $50 \%(\mathrm{p}=0.019)$, GAP stage III $(\mathrm{p}=0.034)$, and BAL recovery rates $<30 \%(p<0.001)$ were the significant risk factors for post-BAL AE (Table 3). 
Table 2. Clinical parameters of the AE group and non-AE group

\begin{tabular}{|c|c|c|c|}
\hline & $\operatorname{AE}[n=5]$ & non-AE [n = 150] & $P$ value \\
\hline Age [years] & $70.0 \pm 5.2$ & $68.6 \pm 7.1$ & 0.78 \\
\hline HRCT diagnosis (UIP/probable UIP/indeterminate for UIP) & $5 / 0 / 0$ & $63 / 56 / 30$ & 0.14 \\
\hline Confidence level of IPF (confident/high confidence/low confidence/unclassifiable) & $5 / 0 / 0 / 0$ & $65 / 49 / 20 / 16$ & 0.14 \\
\hline $\mathrm{PaO}_{2}[\mathrm{~mm} \mathrm{Hg}]$ & $68.6 \pm 15.1$ & $80.9 \pm 12.2$ & 0.062 \\
\hline $\mathrm{KL}-6[\mathrm{U} / \mathrm{mL}]$ & $1068 \pm 258$ & $1363 \pm 1219$ & 0.75 \\
\hline $\mathrm{CRP}[\mathrm{mg} / \mathrm{dL}]$ & $1.8 \pm 3.7$ & $0.4 \pm 0.8$ & 0.10 \\
\hline$\% F V C[\%]$ & $70.4 \pm 24.2$ & $75.8 \pm 17.7$ & 0.36 \\
\hline$\% D_{\text {LCO }}[\%]$ & $39.6 \pm 9.1$ & $67.9 \pm 23.1$ & 0.0074 \\
\hline GAP stage $[\mathrm{I} / \mathrm{II} / \mathrm{III}]$ & $0 / 3 / 2$ & $87 / 51 / 12$ & 0.0086 \\
\hline TCC in BAL $\left[10^{5} / \mathrm{mm}^{3}\right]$ & $1.8 \pm 1.0$ & $2.4 \pm 2.0$ & 0.62 \\
\hline Neutrophils in BAL [\%] & $13.1 \pm 10.5$ & $4.3 \pm 8.6$ & 0.0059 \\
\hline Lymphocytes in BAL [\%] & $8.3 \pm 8.9$ & $18.5 \pm 17.3$ & 0.086 \\
\hline Recovery rates in BAL [\%] & $35.2 \pm 17.6$ & $52.0 \pm 13.4$ & 0.029 \\
\hline TBLB, n [\%] & $4[80 \%]$ & 127 [85\%] & 0.57 \\
\hline
\end{tabular}

Data are expressed as mean \pm standard deviation. AE — acute exacerbation; HRCT — high-resolution computed tomography; UIP — usual interstitial pneumonia; IPF — idiopathic pulmonary fibrosis; KL-6 — Krebs von den Lungen-6; CRP — C-reactive protein; FVC — forced vital capacity; $D_{L C o}$ - diffusing capacity of the lungs for carbon monoxide; GAP stage — Gender-Age-Physiology stage; TCC — total cell counts; BAL — bronchoalveolar lavage; TBLB — transbronchial lung biopsy

Table 3. Univariate logistic regression analysis of risk of acute exacerbation within $\mathbf{3 0}$ days post-bronchoalveolar lavage

\begin{tabular}{|c|c|c|c|c|}
\hline & & HR & $95 \% \mathrm{Cl}$ & $P$ value \\
\hline \multirow[t]{2}{*}{ Age [years] } & $\geq 65$ & 1.41 & $0.15-13.0$ & 0.76 \\
\hline & $>65$ & Ref & & \\
\hline \multirow[t]{2}{*}{$\mathrm{PaO}_{2}[\mathrm{~mm} \mathrm{Hg}]$} & $\geq 75$ & 0.094 & $0.01-0.86$ & 0.036 \\
\hline & $<75$ & Ref & & \\
\hline \multirow[t]{2}{*}{$\mathrm{KL}-6$ [U/mL] } & $\geq 800$ & 2.21 & $0.24-20.3$ & 0.48 \\
\hline & $>800$ & Ref & & \\
\hline \multirow[t]{2}{*}{$\mathrm{CRP}[\mathrm{mg} / \mathrm{dL}]$} & $\geq 0.3$ & 1.42 & $0.22-8.78$ & 0.70 \\
\hline & $<0.3$ & Ref & & \\
\hline \multirow{2}{*}{ \%FVC [\%] } & $\geq 60$ & 0.40 & $0.06-2.55$ & 0.33 \\
\hline & $<60$ & Ref & & \\
\hline \multirow[t]{2}{*}{$\% \mathrm{D}_{\text {Lco }}[\%]$} & $\geq 50$ & 0.069 & $0.007-0.64$ & 0.019 \\
\hline & $<50$ & Ref & & \\
\hline \multirow[t]{2}{*}{ GAP stage } & III & 7.67 & $1.17-50.4$ & 0.034 \\
\hline & $1 / 11$ & Ref & & \\
\hline \multirow[t]{2}{*}{ TCC $\left[10^{5} / \mathrm{mm}^{3}\right]$} & $\geq 2.0$ & 0.22 & $0.025-2.10$ & 0.19 \\
\hline & $<2.0$ & Ref & & \\
\hline \multirow[t]{2}{*}{ Neutrophils in BAL [\%] } & $\geq 7$ & 22.9 & $2.43-215$ & 0.0061 \\
\hline & $<7$ & Ref & & \\
\hline \multirow[t]{2}{*}{ Lymphocytes in BAL [\%] } & $\geq 6$ & 0.070 & $0.007-0.65$ & 0.019 \\
\hline & $<6$ & Ref & & \\
\hline \multirow[t]{2}{*}{ Recovery rates in BAL [\%] } & $\geq 30$ & 0.037 & $0.005-0.25$ & $<0.001$ \\
\hline & $<30$ & Ref & & \\
\hline
\end{tabular}


Multivariate analysis was difficult to perform because the number of events was as small as five.

From the PubMed and J-STAGE databases, we discovered 12 cases from five reports that evaluated BAL-induced AE-IPF (Table 4) [8, 14-17]. All five of our cases improved and survived with systemic steroid therapy. Although the BAL recovery rates were sometimes inadequate, the average BAL recovery rate of the previous and present studies was $48 \%$ (range: 17-80\%). Additionally, the average predicted \%FVC was $69.5 \%$ (range: $42.5-99 \%$ ), but the average $\% \mathrm{D}_{\mathrm{LCO}}$ was $45 \%$ (range: 16-81\%). Furthermore, the C-reactive protein (CRP) level and/or neutrophil count in the BAL fluid were increased in a few cases.

\section{Discussion and conclusions}

In this study, we investigated the risk factors for BAL-induced AE-IPF. Our analyses revealed several risk factors for BAL-induced AE-IPF: (1) neutrophils in $\mathrm{BAL} \geq 7 \%$, which is an indicator of inflammation and instability in the lung, (2) the BAL recovery rate, which has not been reported as a risk factor previously, and (3) disease severity $\left(\mathrm{PaO}_{2}<75 \mathrm{~mm} \mathrm{Hg}, \% \mathrm{D}_{\mathrm{LCO}}<50 \%\right.$, or GAP stage III). We first revealed that the BAL procedure itself was involved in the development of $\mathrm{AE}$ after the procedure.

Atkins et al. [18] reported that the incidence of $\mathrm{AE}$ was 4.1 per 100 patient-years based on a meta-analysis from six clinical trials. Additionally, in the INPULSIS trial, a phase III randomized trial of nintedanib ( $\mathrm{n}=1066)$, Richeldi et al. [19] reported that AE-IPF occurred within 1 year in $7.6 \%$ of patients who received a placebo. Among the 155 patients in our study, the incidence of $\mathrm{AE}$ within the first 30 days after the BAL procedure was significantly higher than that over the entire observation period (first 30 days: 3.2 per 100 patients-30 days; entire observation period: 0.36 per 100 patients-30 days, $p<0.001$ ), suggesting a risk of $\mathrm{AE}$ in patients with IPF for at least 30 days after BAL.

The high neutrophil level in BAL $(\geq 7 \%)$ was a significant risk factor for AE after BAL (HR, 17.6; 95\% CI, 1.17-265; $p=0.038$; Table 3). The subjects with elevated neutrophil counts, as indicated by BAL, were predicted to have had increased lung activity at the time BAL was performed. This informed our conclusion that $\mathrm{AE}$ was likely to occur in these patients. Kinder et al. [20] reported increased neutrophils in BAL fluid to be an independent predictor of early mortality among patients with IPF. Sakamoto et al. [8] focused on the disease's "instability", an elevated CRP level (>1 mg/dL) and/or increased white blood cell count ( $>9000 / \mathrm{mm}^{3}$ ), which is unusual for stable IPF cases, were observed in six cases. Careful follow-up is important in patients with "instability," but a high neutrophil level in BAL is a factor unknown before a BAL procedure and thus is not suitable for predicting the onset of BAL-induced AE.

We showed that a low BAL fluid recovery rate was a significant risk factor for $\mathrm{AE}$ after $\mathrm{BAL}$ (HR, 0.89; 95\% CI, 0.81-0.97; p = 0.012; Table 3). Ogata et al. [21] reported that the frequency of complications (mainly hypoxemia and fever, not only AE) after BAL increased if the BAL recovery rate was poor. Although the study conducted by Ogata et al. [21] included various diffuse lung diseases [i.e., interstitial lung disease (20.7\%) and pathologies, including IPF (no description), sarcoidosis (23.9\%), infection (11.6\%), collagen vascular disease-associated interstitial pneumonia (9.8\%), and drug-induced interstitial pneumonia (9.8\%)], our findings were consistent with those of their report. Poor BAL fluid recovery implies that much of the saline used for lavage remained in the lungs after the procedure. Saline is thought to be naturally absorbed into the blood vessels in the alveoli. If excessive saline remains in the alveoli, infection is promoted, and it is possible that $\mathrm{AE}$ occurs due to lung infection. Some reports have stated that saline lavage per se may cause lung injury. In an animal model, repeated BAL with saline resulted in acute lung injury [22, 23]. Matute-Bello et al. [23] reported that repeated lavage with saline reduced the surfactant lipid concentration in alveolar lining fluids and ultimately altered alveolar surface tension. Decreasing the surfactant causes lung injury by facilitating alveolar collapse, increasing mechanical injury, and impairing alveolar host defenses, a likely finding on both animal models and actual patients with IPF.

We found that disease severity $\left(\mathrm{PaO}_{2}<\right.$ $75 \mathrm{~mm} \mathrm{Hg}, \% \mathrm{D}_{\mathrm{LCO}}<50 \%$, or GAP stage III) was a significant risk factor for AE after BAL only in the univariate analysis. Several risk factors for the development of AE, including low \%FVC [24-28], low $\mathrm{D}_{\mathrm{LCO}}$ [24-26], and poor baseline oxygenation [24], have been previously identified. In a review of 12 case reports, Sakamoto et al. [8] reported that the severity of IPF before the BAL procedure was moderate to severe in patients who met any of the following criteria: (1) \%FVC $<65 \%$, (2) desaturation with exertion, and (3) $\mathrm{D}_{\mathrm{LCO}} \leq 50 \%$. The findings support our results and indicate that 


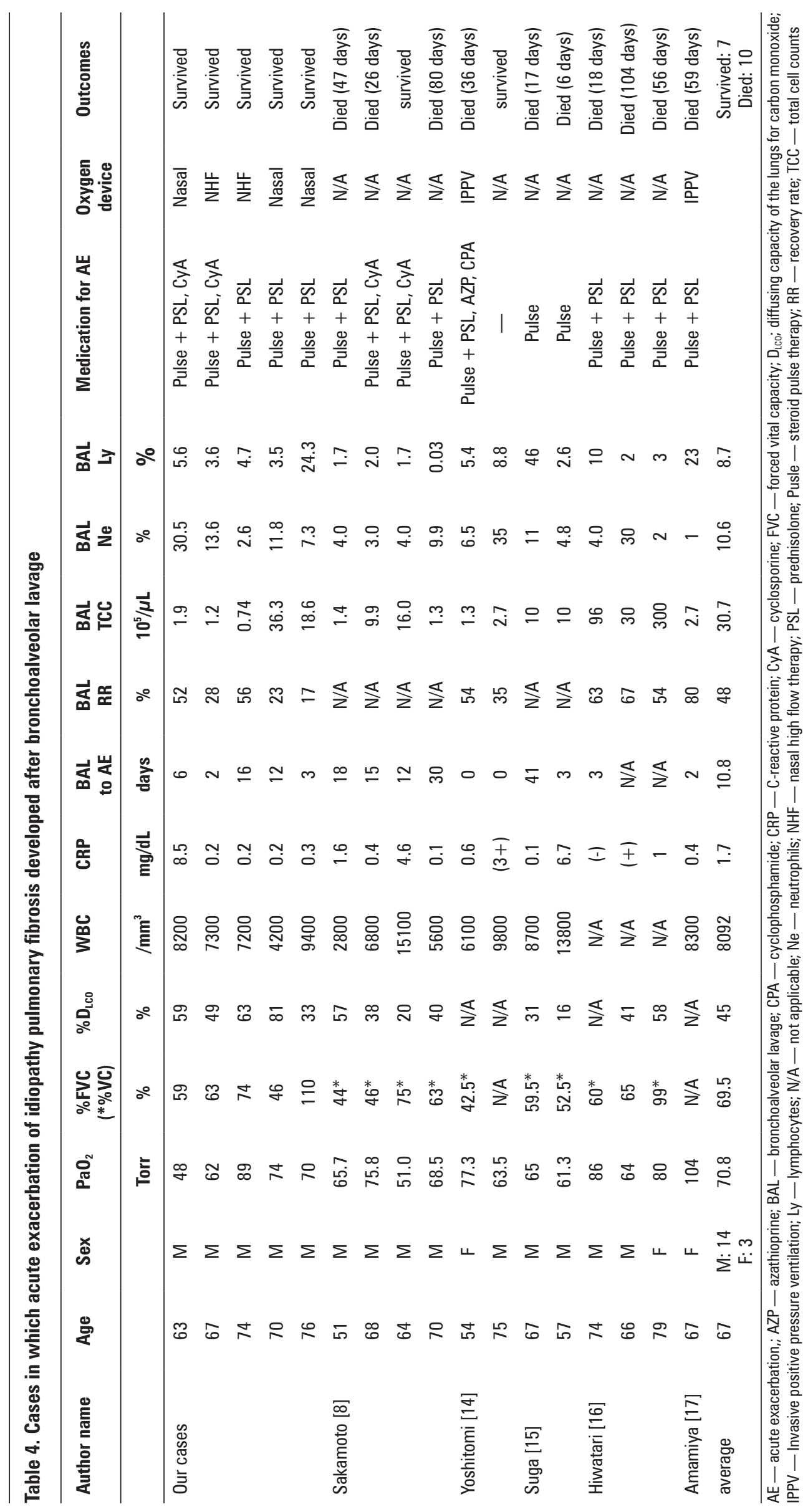


some patients are already susceptible to AEs at the time of BAL.

We conducted a literature review, but there were no reports of BAL-induced AE since Sakamoto's analysis in 2012 [8]. One reason might be publication bias. Since BAL-induced AE is a major significant complication, it is necessary to reduce the publication bias to evaluate the risk. Our study revealed that the first performance of BAL might cause AE. By contrast, Sakamoto et al. [8] reported that the first BAL did not induce AE in any of four cases with $\mathrm{AE}$ that had undergone at least one previous BAL.

According to Sakamoto et al. [8] only one case survived the AE. In the current study, all five cases were successfully discharged. The death of 10 of the 12 patients reported in the literature (Table 4.) underscores the necessity of addressing AEs due to BAL. The therapies that we administered to the five patients with AEs in our study were similar to those administered to the subjects in the study conducted by Sakamoto et al. [8]. These therapies are outlined in Table 4. Additionally, advances in oxygen therapy and medical treatment may have contributed to improved mortality in our cases. Vianello et al. [29] evaluated the utility of a highflow nasal cannula for patients with AE-IPF who did not respond to treatment using a conventional nasal cannula. In our cases, a high-flow nasal cannula was used in two cases. It is not possible to confirm whether the cases we reviewed used a high-flow nasal cannula.

This study's AE incidence (5 in 155 cases, $3.2 \%$ ) is higher than in previous reports (4 in 202 cases, 1.9\% [8]; none of 57 cases, 0\% [21]; and two in 104 cases, $1.9 \%$ [15]). Although no detailed patient background was reported by Sakamoto et al. [8], the average age was 64 years, which was younger than that in our study (68.6 years). In addition, a surgical lung biopsy was performed in $47.3 \%$ of cases, suggesting that there are many relatively mild cases in which surgical lung biopsy is possible. Regarding Ogata's report [21], there is no patient background limited to idiopathic interstitial pneumonias, but the overall average age is as young as 58.7 years. These findings are in line with our findings that high severity is a risk factor for AEs. In Suga's report [15], the overall patient background is not mentioned.

Our study had some limitations. First, this was a single-center retrospective study with a small number of cases. Only five patients developed AE after BAL. Since the sample size was small, further studies with more patients are required. Second, the effects of the type of fiber used in bronchoscopy, years of experience of the examiner, examination time, and other bronchoscopic procedures on the development of AEs were not analyzed in the current study. Finally, BAL may not have been the cause of AE in some cases. An increase in the neutrophil level in BAL $(\geq 7 \%$ ) was confirmed in four cases. These results suggest that latent infection or disease progression may have existed before BAL.

\section{Clinical implications/future directions}

In conclusion, patients with suspected IPF may develop AE after BAL and should be monitored carefully. Disease severity, high neutrophil levels in BAL, and poor BAL recovery rates may be risk factors for BAL-induced AEs and need to be confirmed in a larger, multi-center prospective study. We will be able to assess some administration parameters, such as the number of years of experience of the examiner and examination times. Other factors to consider are the lavage equipment and its components.

\section{Acknowledgements}

We thank Mai Fujie, our medical engineer, for supporting our BAL examination. We would also like to thank Editage (www.editage.jp) for English language editing.

\section{Source of funding}

This work was supported by a Japan Society for the Promotion of Science KAKENHI Grant (number: JP19K20727).

\section{Conflict of interest}

None declared.

\section{References:}

1. Wells AU. The clinical utility of bronchoalveolar lavage in diffuse parenchymal lung disease. Eur Respir Rev. 2010; 19(117): 237-241, doi: 10.1183/09059180.00005510, indexed in Pubmed: 20956199.

2. Gharsalli H, Mlika M, Sahnoun I, et al. The utility of bronchoalveolar lavage in the evaluation of interstitial lung diseases: A clinicopathological perspective. Semin Diagn Pathol. 2018; 35(5): 280-287, doi: 10.1053/i.semdp.2018.08.003, indexed in Pubmed: 30173880.

3. Meyer KC, Raghu G, Baughman RP, et al. American Thoracic Society Committee on BAL in Interstitial Lung Disease. An official American Thoracic Society clinical practice guideline: the clinical utility of bronchoalveolar lavage cellular analysis in interstitial lung disease. Am J Respir Crit Care Med. 2012; 185(9): 1004-1014, doi: 10.1164/rccm.201202-0320ST, indexed in Pubmed: 22550210.

4. Ohshimo S, Bonella F, Cui Ai, et al. Significance of bronchoalveolar lavage for the diagnosis of idiopathic pulmonary fibrosis. Am J Respir Crit Care Med. 2009; 179(11): 1043-1047, doi: 10.1164/rccm.200808-1313OC, indexed in Pubmed: 19246718. 
5. Raghu G, Collard HR, Egan JJ, et al. ATS/ERS/JRS/ALAT Committee on Idiopathic Pulmonary Fibrosis. An official ATS/ ERS/JRS/ALAT statement: idiopathic pulmonary fibrosis: evidence-based guidelines for diagnosis and management. Am J Respir Crit Care Med. 2011; 183(6): 788-824, doi: 10.1164/ rccm.2009-040GL, indexed in Pubmed: 21471066.

6. Raghu G, Remy-Jardin M, Ryerson CJ, et al. Diagnosis of Hypersensitivity Pneumonitis in Adults. An Official ATS/JRS/ALAT Clinical Practice Guideline. Am J Respir Crit Care Med. 2020; 202(3): e36-e69, doi: 10.1164/rccm.202005-2032ST, indexed in Pubmed: 32706311.

7. Raghu G, Remy-Jardin M, Myers JL, et al. American Thoracic Society, European Respiratory Society, Japanese Respiratory Society, and Latin American Thoracic Society. Diagnosis of Idiopathic Pulmonary Fibrosis. An Official ATS/ERS/JRS/ALAT Clinical Practice Guideline. Am J Respir Crit Care Med. 2018; 198(5): e44-e68, doi: 10.1164/rccm.201807-1255ST, indexed in Pubmed: 30168753.

8. Sakamoto K, Taniguchi H, Kondoh Y, et al. Acute exacerbation of IPF following diagnostic bronchoalveolar lavage procedures. Respir Med. 2012; 106(3): 436-442, doi: 10.1016/j. rmed.2011.11.006, indexed in Pubmed: 22138357.

9. Amundson WH, Racila E, Allen T, et al. Acute exacerbation of interstitial lung disease after procedures. Respir Med. 2019; 150: 30-37, doi: 10.1016/j.rmed.2019.02.012, indexed in Pubmed: 30961948.

10. Ryerson CJ, Corte TJ, Lee JS, et al. A Standardized Diagnostic Ontology for Fibrotic Interstitial Lung Disease. An International Working Group Perspective. Am J Respir Crit Care Med. 2017; 196(10): 1249-1254, doi: 10.1164/rccm.201702-0400PP, indexed in Pubmed: 28414524.

11. Ley B, Ryerson CJ, Vittinghoff E, et al. A multidimensional index and staging system for idiopathic pulmonary fibrosis. Ann Intern Med. 2012; 156(10): 684-691, doi: 10.7326/0003-4819156-10-201205150-00004, indexed in Pubmed: 22586007.

12. Collard HR, Ryerson CJ, Corte TJ, et al. Acute Exacerbation of Idiopathic Pulmonary Fibrosis. An International Working Group Report. Am J Respir Crit Care Med. 2016; 194(3): 265-275, doi: 10.1164/rccm.201604-0801CI, indexed in Pubmed: 27299520.

13. Kanda Y. Investigation of the freely available easy-to-use software 'EZR' for medical statistics. Bone Marrow Transplant. 2013; 48(3): 452-458, doi: 10.1038/bmt.2012.244, indexed in Pubmed: 23208313.

14. Yoshitomi A, Sato A, Tamura R, et al. [Acute exacerbation following bronchoalveolar lavage in idiopathic interstitial pneumonia]. Nihon Kyobu Shikkan Gakkai Zasshi. 1993; 31(12): 1606-1611, indexed in Pubmed: 8121101.

15. Suga T, Sugiyama Y, Ohno S, et al. [Two cases of IIP which developed acute exacerbation after bronchoalveolar lavage]. Nihon Kyobu Shikkan Gakkai Zasshi. 1994; 32(2): 174-178, indexed in Pubmed: 8164406.

16. Hiwatari N, Shimura S, Takishima T, et al. Bronchoalveolar lavage as a possible cause of acute exacerbation in idiopathic pulmonary fibrosis patients. Tohoku J Exp Med. 1994; 174(4): 379-386, doi: 10.1620/tjem.174.379, indexed in Pubmed: $\underline{7732520 .}$.

17. Amamiya T, Nishi K, Mizuguchi M, et al. A case of acute type idiopathic interstitial pneumonia associated with acute exacerbation. J Jpn Soc Respir Endoscopy. 1995; 17(3-4): 80-86.

18. Atkins CP, Loke YK, Wilson AM. Outcomes in idiopathic pulmonary fibrosis: a meta-analysis from placebo controlled trials. Respir Med. 2014; 108(2): 376-387, doi: 10.1016/j. rmed.2013.11.007, indexed in Pubmed: 24440032.

19. Richeldi L, du Bois RM, Raghu G. Efficacy and safety of nintedanib in idiopathic pulmonary fibrosis. N Engl J Med. 2014; 370(22): 2071-2082, doi: 10.1056/NEJMoa1402584.

20. Kinder BW, Brown KK, Schwarz MI, et al. Baseline BAL neutrophilia predicts early mortality in idiopathic pulmonary fibrosis. Chest. 2008; 133(1): 226-232, doi: 10.1378/chest.07-1948, indexed in Pubmed: 18071016.

21. Ogata-Suetsugu S, Hamada N, Mikumo N, et al. Complications of bronchoalveolar lavage (BAL) at a single institution. J Jpn Soc Respir Endoscopy. 2016; 38(3): 173-178.

22. Lachmann B, Robertson B, Vogel J. In vivo lung lavage as an experimental model of the respiratory distress syndrome. Acta Anaesthesiol Scand. 1980; 24(3): 231-236, doi: 10.1111/j.13996576.1980.tb01541.x, indexed in Pubmed: 7445941.

23. Matute-Bello G, Frevert CW, Martin TR. Animal models of acute lung injury. Am J Physiol Lung Cell Mol Physiol. 2008; 295(3): L379-L399, doi: 10.1152/ajplung.00010.2008, indexed in Pubmed: 18621912.

24. Collard HR, Yow E, Richeldi L, et al. IPFnet investigators. Suspected acute exacerbation of idiopathic pulmonary fibrosis as an outcome measure in clinical trials. Respir Res. 2013; 14: 73, doi: 10.1186/1465-9921-14-73, indexed in Pubmed: 23848435.

25. Ohshimo S, Ishikawa N, Horimasu Y, et al. Baseline KL-6 predicts increased risk for acute exacerbation of idiopathic pulmonary fibrosis. Respir Med. 2014; 108(7): 1031-1039, doi: 10.1016/j.rmed.2014.04.009, indexed in Pubmed: 24835074.

26. Song JW, Hong SB, Lim CM, et al. Acute exacerbation of idiopathic pulmonary fibrosis: incidence, risk factors and outcome. Eur Respir J. 2011; 37(2): 356-363, doi: 10.1183/09031936.00159709, indexed in Pubmed: 20595144.

27. Kondoh Y, Taniguchi H, Katsuta T. Risk factors of acute exacerbation of idiopathic pulmonary fibrosis. Sarcoidosis Vasc Diffuse Lung Dis. 2010; 27(2): 103-110, indexed in Pubmed: 21319592.

28. Kishaba T, Tamaki H, Shimaoka Y, et al. Staging of acute exacerbation in patients with idiopathic pulmonary fibrosis. Lung. 2014; 192(1): 141-149, doi: 10.1007/s00408-013-9530-0, indexed in Pubmed: 24221341.

29. Vianello A, Arcaro G, Molena B, et al. High-flow nasal cannula oxygen therapy to treat acute respiratory failure in patients with acute exacerbation of idiopathic pulmonary fibrosis. Ther Adv Respir Dis. 2019; 13: 1753466619847130, doi: 10.1177/1753466619847130, indexed in Pubmed: 31170875. 